\title{
Optimal Control for maximizing Link Velocity of Robotic Variable Stiffness Joints
}

\author{
Sami Haddadin, Michael Weis, Sebastian Wolf, and \\ Alin Albu-Schäffer \\ Institute of Robotics and Mechatronics, DLR - German Aerospace \\ Center, Wessling, Germany (Tel: +49(0)8153 28 1047; e-mail: \\ sami.haddadin@dlr.de, alin.albu-schaeffer@dlr.de, \\ sebastian.wolf@dlr.de).
}

\begin{abstract}
In this paper we evaluate the potential of Variable Stiffness Actuation to utilize its inherent joint elasticity and capability to adjust the intrinsic joint stiffness. These abilities make it possible to realize fundamentally different motion control schemes in comparison to intrinsically stiff robots. In this paper we treat the problem of how to generate optimally fast link side velocity at a certain time instant by fully exploiting the elastic energy transfer effects between motor, joint elasticity, stiffness adjustment mechanism, and link. Based on optimal control theory we show that it is possible to significantly and optimally exceed the motor maximum velocity by appropriate motor commands. We solve the problem for models of increasing complexity in order to consecutively elaborate the core insights into the chosen problem. Finally, we present experimental results with a VIA joint prototype, confirming the correctness of the developed formalism.
\end{abstract}

\section{INTRODUCTION}

Classical articulated robots are characterized by stiff actuation with elastic effects being certainly unwanted. Position accuracy and repeatability are the goals that are aimed for. If compliance is desired it is realized via active control, leading to such sophisticated solutions as for the DLR Lightweight Robot III, Albu-Schäffer et al. [2007]. The robot utilizes integrated joint torque sensors to realize e.g. high-performance Cartesian impedance control. However, recently elastic joints received increasing attention as several interesting properties are achieved, if significant intrinsic compliance is incorporated into the design. A general argument in favor of intrinsic joint compliance, apart from its role for joint protection from impact shocks, is its ability to store and release energy

(1) for decreasing the energy consumption of the system or

(2) to increase peak power output.

The former has received larger attention especially for biped walking Yamaguchi et al. [1998a,b], Vanderborght et al. [2006]. Our focus, however, lies on the latter as it allows to considerably increase the link speed Schempf et al. [1995], Paluska and Herr [2006], Okada et al. [2002], Haddadin et al. [2007], Wolf and Hirzinger [2008] above motor speed level. In most cases constant joint elasticity is used (Series Elastic Actuation (SEA)), however, recently also the concept of Variable Impedance Actuation (VIA), which can be considered as an extension of SEA has drawn large attention. The principle of VIA is truly human-inspired in the sense that it intends to approach the impedance adjustment capabilities of the human musculoskeletal system. In humans all muscles work in pairs, namely the agonist and the antagonist. For transferring

\footnotetext{
$\star$ This work has been partially funded by the European Commission's Sixth Framework Programme as part of the project VIACTORS under grant no. 231554.
}

this design idea to robotic actuation there are numerous concrete concepts. An overview is e.g. given in van Ham et al. [2009]. At DLR we developed an integrated hand arm system, Grebenstein and van der Smagt [2008], AlbuSchäffer et al. [2008] that is fully equipped with variable impedance actuation, c.f. Fig.1.

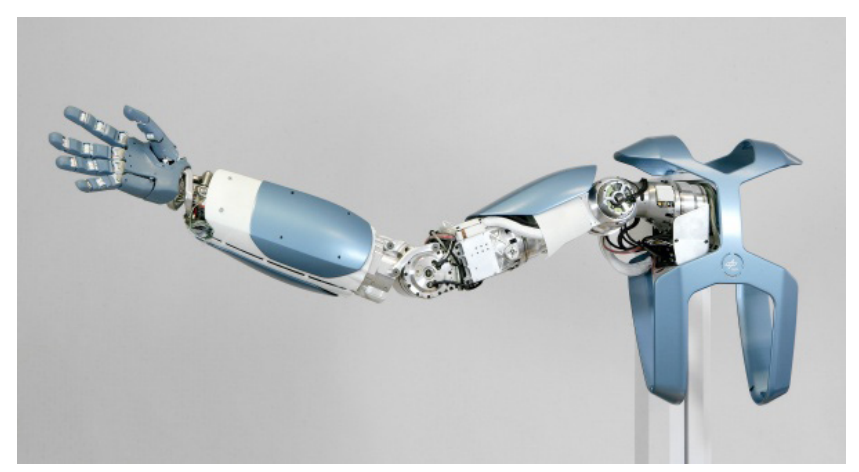

Fig. 1. The DLR hand arm system.

Their unique characteristic is an intrinsically variable impedance element between actuator and link, c.f. Fig 2.

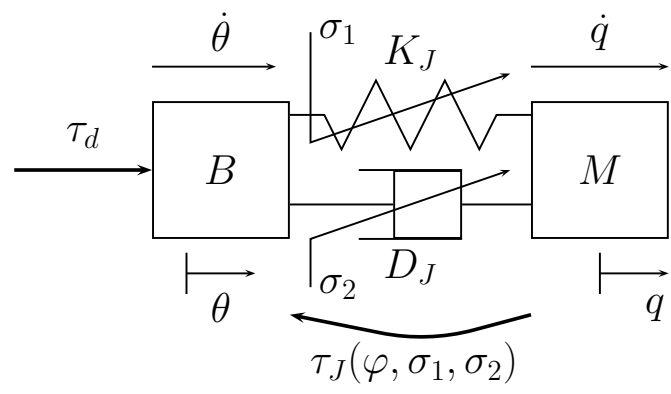

Fig. 2. 1-DoF model of a VIA joint. 
The elastic joint torque $\tau_{J}\left(\varphi, \sigma_{1}, \sigma_{2}\right)$ between motor inertia $B$ (associated with motor position $\theta$ ) and link inertia $M$ (associated with link position $q$ ) is in general a function of the elastic deflection $\varphi=\theta-q$, as well as of the stiffness and damper actuation variables $\sigma_{1}, \sigma_{2}$. The desired motor torque is denoted as $\tau_{d}$.

As the mechanical complexity and capabilities of such joints are significantly different from classical stiff ones, there are still numerous open problems. One of the most remarkable properties of VIA is that the elastic joint element can be used to store and release energy. It is therefore fundamental to analyze how this property can be used for generating motions that take advantage of this and significantly enhance the capabilities of VIA robots in comparison with their stiff counterparts. Recently, it was shown experimentally that it is possible to design VIA motions such that the link side velocity can significantly exceed the maximum motor velocity, Wolf and Hirzinger [2008]. This is especially useful for achieving human like peak performance by means of maximum speed ${ }^{1}$.

In this paper we develop the theory to maximize the link side velocity of a variable impedance joint and verify the results experimentally. For solving this problem, we use methods from optimal control theory. In order to systematically analyze the different effects and constraints we increase the complexity of the used models and try to find analytical solutions if possible. Table 1 depicts the consecutive steps we have made and points out whether analytical or numerical solutions were obtained. First, we solve the constant stiffness case (case A) with different motor models (case $\mathrm{B}+\mathrm{C}$ ), incorporate the presence of bounds on the state variables (case D), analyze the influence of adjusting the stiffness (case $F+G$ ), and finally discuss experimental results on the DLR QA-Joint (case $\mathrm{H})$. Each step contributes particular insights, as e.g. the influence of constrained motor dynamics, constraints on the elastic deflection, or stiffness adjustment, which makes it possible to formulate a full view on the problem. As mechanical damping is usually unwanted due to energetic arguments, most VIA implementations realize damping via active control and not through a mechanically complex solution. Therefore, we do not consider damping in this paper, i.e. $D_{J}=0$. Furthermore, we assume $K_{J}=\sigma_{1}$ for the theoretical analysis in order to keep it clear for the reader. Therefore, we use only $\sigma$ to denote the stiffness actuation variable from now on.

\section{PROBLEM FORMULATION}

As we assume systems which state space equations do not explicitly depend on time, the description of their dynamics is a system of differential equations of first order.

$$
\dot{\mathbf{x}}(t)=f(\mathbf{x}(t), \mathbf{u}(t))
$$

with $\mathbf{x}$ and $\mathbf{u}$ being the state vector and control input, respectively. For achieving an optimal control input, a general optimality criterion is usually to be chosen such that the timely evolution of $\mathbf{x}(t)$ and $\mathbf{u}(t)$, as well as the final state of the system $\mathbf{x}\left(t_{f}\right)$ are weighted with respect to each other. Therefore, an integral cost functional is a reasonable choice, as it weights the final state with the

\footnotetext{
1 Extreme examples show that humans are capable of generating enormous joint speeds as e.g. shoulder rotation of $6.900-9800 \% / \mathrm{s}$ during a baseball pitch of a professional pitcher Herman [2007]. This speed range is currently not realizable by robots if the torque range and the weight of the joint should be also compatible with human values.
}

function $h$ and the timely evolution of the state and control input with integrating the function $g$.

$$
J=h\left(\mathbf{x}\left(t_{f}\right), t_{f}\right)+\int_{0}^{t_{f}} g(\mathbf{x}(t), \mathbf{u}(t), t) \mathrm{d} t
$$

Together with the Hamiltonian

$$
H(\mathbf{x}(t), \boldsymbol{\lambda}(t), \mathbf{u}(t), t)=-g(\mathbf{x}(t), \mathbf{u}(t), t)+\boldsymbol{\lambda}^{T} f(\mathbf{x}(t), \mathbf{u}(t), t)(3)
$$

the constrained optimization problem is transformed into a problem without constraints. However, in order to maximize the link side velocity at a certain time instant $t_{f}$ only, (2) reduces to:

$$
\left.J=h\left(\mathbf{x}\left(t_{f}\right), t_{f}\right)\right)=\dot{q}\left(t_{f}\right)
$$

Since no other constraints are taken into consideration (3) reduces to

$$
H(\mathbf{x}, \boldsymbol{\lambda}, u, t)=\boldsymbol{\lambda}^{T} f(\mathbf{x}(t), u(t), t) .
$$

For the optimization of the final state the boundary conditions of the adjoint equations result from the transversal condition

$$
\boldsymbol{\lambda}\left(t_{f}\right)=\frac{\partial h\left(t_{f}\right)}{\partial \mathbf{x}}
$$

Together with the initial boundary conditions of the state space equation and the final boundary conditions of the adjoint equations lead to a two point boundary problem. The partial derivatives of the Hamiltonian with regard to the state and co-states define a canonical system of differential equations that needs to be solved:

$$
\begin{array}{r}
\dot{\mathbf{x}}=\frac{\partial H}{\partial \boldsymbol{\lambda}} \\
\dot{\boldsymbol{\lambda}}=-\frac{\partial H}{\partial \mathbf{x}}
\end{array}
$$

In the next section we analyze models of increasing complexity in order to elaborate the fundamental aspects about optimizing the link side velocity at a certain time instant $t_{f}$.

\section{OPTIMAL CONTROL FOR LINEAR CASES}

In this section we treat the constant elasticity case $\left(K_{J}=\right.$ const.). Stiffness adjustment and other nonlinear effects are discussed in Sec. 4 and Sec. 5. For the first model the motor behaves as a velocity source, which gives insight into the principles of utilizing joint elasticity. In order to investigate the influence of motor dynamics on the switching trajectory, we then consider the motor to be position controlled. We investigate both PT1 and PT2 behavior for the controlled motor. In a first step we neglect the influence of the elastic joint torque feedback on the motor inertia as this allows to find a closed solution ${ }^{2}$. Finally, the feedback of the elastic joint torque is also considered. The actuating variable $u$ is chosen to be the desired motor speed $\theta_{d}$. The proportional and damping gain values for the motor controller are denoted as $K_{P}$ and $K_{D}$, respectively.

\footnotetext{
2 Please note that the stiffness of the motor PD controller is three order of magnitudes larger than the joint stiffness. Therefore, the effect of the elastic torque is expected to be reasonably small to neglect this effect. This will be later on confirmed with realistic simulation parameters.
} 


\begin{tabular}{l|l|l|l|} 
case & model & solution & achieved insights \\
\hline \hline A & Velocity source + SEA & analytical & principal effect of significant joint elasticity \\
\hline B & PT1 + SEA & analytical & influence of constrained motor dynamics, 1st order \\
\hline C & PT2 + SEA & analytical & influence of constrained motor dynamics, 2nd order \\
\hline D & PT2 + SEA + JTF & numerical & influence of joint torque feedback on motor inertia \\
\hline E & PT2 + SEA + JTF + CD & numerical & influence of deflection constraints \\
\hline F & Velocity source + VS & analytical & principle effect of stiffness adjustment \\
\hline G & Velocity source + VS + CD & numerical & influence of stiffness adjustment and constrained deflection \\
\hline H & PT2 + VS + CMT & numerical & real VIA design behavior and constrained motor torque \\
\hline
\end{tabular}

Table 1. Analyzed models $(\mathrm{SEA}=$ Series Elastic Actuation, JTF = joint torque feedback, CD $=$ constrained deflection, $\mathrm{VS}=$ variable stiffness, $\mathrm{CMT}=$ constrained motor torque).

\begin{tabular}{|c|c|c|c|c|}
\hline & Vel. source (A) & $\mathrm{PT} 1(\mathrm{~B})$ & $\mathrm{PT} 2(\mathrm{C})$ & $\mathrm{PT} 2+\tau_{J}(\mathrm{D})$ \\
\hline 1 & $\begin{aligned} \theta & =\int_{0}^{t_{f}} \dot{\theta}_{d} \mathrm{~d} t \\
M \ddot{q} & =K_{J}(\theta-q)\end{aligned}$ & $\begin{aligned} \tau_{m} & =K_{P}\left(\dot{\theta}_{d}-\dot{\theta}\right) \\
\tau_{m} & =B \ddot{\theta} \\
M \ddot{q} & =K_{J}(\theta-q)\end{aligned}$ & $\begin{aligned} \tau_{m} & =K_{D}\left(\dot{\theta}_{d}-\dot{\theta}\right)+K_{P}\left(\theta_{d}-\theta\right) \\
\tau_{m} & =B \ddot{\theta} \\
M \ddot{q} & =K_{J}(\theta-q)\end{aligned}$ & $\begin{aligned} \tau_{m} & =K_{D}\left(\dot{\theta}_{d}-\dot{\theta}\right)+K_{P}\left(\theta_{d}-\theta\right) \\
\tau_{m} & =B \ddot{\theta}-K_{J}(\theta-q) \\
M \ddot{q} & =K_{J}(\theta-q)\end{aligned}$ \\
\hline 2 & 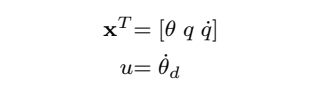 & 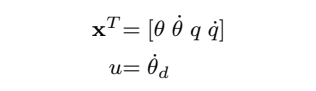 & 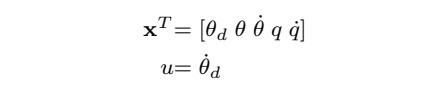 & 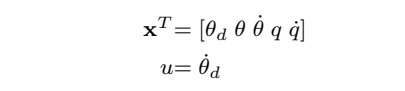 \\
\hline 3 & $\begin{array}{l}\dot{x}_{1}=u \\
\dot{x}_{2}=x_{3} \\
\dot{x}_{3}=\omega^{2}\left(x_{1}-x_{2}\right)\end{array}$ & $\begin{array}{l}\dot{x}_{1}=x_{2} \\
\dot{x}_{2}=\frac{K_{P}}{B}\left(u-x_{2}\right) \\
\dot{x}_{3}=x_{4} \\
\dot{x}_{4}=\frac{K_{J}}{M}\left(x_{1}-x_{3}\right)\end{array}$ & $\begin{array}{l}\dot{x}_{1}=u \\
\dot{x}_{2}=x_{3} \\
\dot{x}_{3}=\frac{1}{B}\left(K_{D}\left(u-x_{3}\right)+\right. \\
\left.\quad+K_{P}\left(x_{1}-x_{2}\right)\right) \\
\dot{x}_{4}=x_{5} \\
\dot{x}_{5}=\frac{K_{J}}{M}\left(x_{2}-x_{4}\right)\end{array}$ & $\begin{array}{l}\dot{x}_{1}=u \\
\dot{x}_{2}=x_{3} \\
\dot{x}_{3}=\frac{1}{B}\left(K_{D}\left(u-x_{3}\right)+\right. \\
\left.\quad+K_{P}\left(x_{1}-x_{2}\right)-K_{J}\left(x_{2}-x_{4}\right)\right) \\
\dot{x}_{4}=x_{5} \\
\dot{x}_{5}=\frac{K_{J}}{M}\left(x_{2}-x_{4}\right)\end{array}$ \\
\hline 4 & $\begin{array}{r}H(\mathbf{x}(t), \boldsymbol{\lambda}(t), u(t), t)= \\
\lambda_{1} u+\lambda_{2} x_{3}+\lambda_{3} \omega^{2}\left(x_{1}-x_{2}\right)\end{array}$ & $\begin{array}{r}H(\mathbf{x}(t), \boldsymbol{\lambda}(t), u(t), t)= \\
\lambda_{1} u+\lambda_{2} x_{3}+\lambda_{3} \omega^{2}\left(x_{1}-x_{2}\right)\end{array}$ & $\begin{array}{r}H(\mathbf{x}(t), \lambda(t), u(t))=\lambda_{1} u+\lambda_{2} x_{3} \\
+\lambda_{3} \frac{1}{B}\left(K_{D}\left(u-x_{3}\right)+K_{P}\left(x_{1}-x_{2}\right)\right)+ \\
+\lambda_{4} x_{5}+\lambda_{5} \frac{K_{J}}{M}\left(x_{2}-x_{4}\right)\end{array}$ & $\begin{array}{r}H(\mathbf{x}(t), \lambda(t), u(t))=\lambda_{1} u+\lambda_{2} x_{3} \\
+\lambda_{3} \frac{1}{B}\left(K_{D}\left(u-x_{3}\right)+\right. \\
\left.+K_{P}\left(x_{1}-x_{2}\right)-K_{J}\left(x_{2}-x_{4}\right)\right)+ \\
+\lambda_{4} x_{5}+\lambda_{5} \frac{K_{J}}{M}\left(x_{2}-x_{4}\right)\end{array}$ \\
\hline 5 & $\begin{array}{l}\dot{\lambda}_{1}=-\lambda_{3} \omega^{2} \\
\dot{\lambda}_{2}=\lambda_{3} \omega^{2} \\
\dot{\lambda}_{3}=-\lambda_{2}\end{array}$ & $\begin{array}{l}\dot{\lambda}_{1}=-\lambda_{4} \omega \\
\dot{\lambda}_{2}=-\lambda_{1}+\frac{K_{P}}{B} \lambda_{2} \\
\dot{\lambda}_{3}=\lambda_{4} \omega \\
\dot{\lambda}_{4}=-\lambda_{3}\end{array}$ & $\begin{array}{l}\dot{\lambda}_{1}=-\lambda_{3} \frac{K_{P}}{B} \\
\dot{\lambda}_{2}=\lambda_{3} \frac{K_{P}}{B}-\lambda_{5} \omega \\
\dot{\lambda}_{3}=-\lambda_{2}+\lambda_{3} \frac{K_{D}}{B} \\
\dot{\lambda}_{4}=\lambda_{5} \omega \\
\dot{\lambda}_{5}=-\lambda_{4}\end{array}$ & $\begin{array}{l}\dot{\lambda}_{1}=-\lambda_{3} \frac{K_{P}}{B} \\
\dot{\lambda}_{2}=\lambda_{3}\left(\frac{K_{P}}{B}+\frac{K_{J}}{B}\right)-\lambda_{5} \omega \\
\dot{\lambda}_{3}=-\lambda_{2}+\lambda_{3} \frac{K_{D}}{B} \\
\dot{\lambda}_{4}=-\left(\lambda_{3}+\lambda_{5}\right) \frac{K_{D}}{M} \\
\dot{\lambda}_{5}=-\lambda_{4}\end{array}$ \\
\hline 6 & $\begin{aligned} \boldsymbol{\lambda}^{T}\left(t_{f}\right) & =\left[\begin{array}{lll}0 & 0 & 1\end{array}\right] \\
\mathbf{x}^{T}(0) & =\left[\begin{array}{lll}0 & 0 & 0\end{array}\right]\end{aligned}$ & 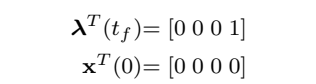 & 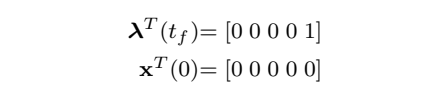 & 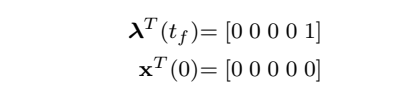 \\
\hline 7 & $\dot{\theta}_{d}^{*}=\left\{\begin{array}{cc}\dot{\theta}_{\max }, & \lambda_{1}>0 \\
\dot{\theta}_{\min }, & \lambda_{1}<0 \\
\text { singular, } & \lambda_{1}=0\end{array}\right.$ & $\dot{\theta}_{d}^{*}=\left\{\begin{array}{cc}\dot{\theta}_{\max }, & \lambda_{2}>0 \\
\dot{\theta}_{\min }, & \lambda_{2}<0 \\
\text { singular, } & \lambda_{2}=0\end{array}\right.$ & $\dot{\theta}_{d}^{*}=\left\{\begin{array}{cc}\dot{\theta}_{\max }, & \lambda_{1}+\frac{K_{D}}{B} \lambda_{3}>0 \\
\dot{\theta}_{\min }, & \lambda_{1}+\frac{K_{D}}{B} \lambda_{3}<0 \\
\text { singular, } & \lambda_{1}+\frac{K_{D}}{B} \lambda_{3}=0\end{array}\right.$ & $\dot{\theta}_{d}^{*}=\left\{\begin{array}{cc}\dot{\theta}_{\max }, & \lambda_{1}+\frac{K_{D}}{B} \lambda_{3}>0 \\
\dot{\theta}_{\min }, & \lambda_{1}+\frac{K_{D}}{B} \lambda_{3}<0 \\
\text { singular, } & \lambda_{1}+\frac{K_{D}}{B} \lambda_{3}=0\end{array}\right.$ \\
\hline
\end{tabular}

Table 2. Summary of the investigated linear optimal control problems.

As the principal approach is always the same we summarized the relevant equations and conditions for the interested reader in Tab. 2 and focus only on the most significant general insights in the following description. Table 2 lists the system dynamics (1), the state and input vector (2), the state space equations (3), the Hamiltonian (4), the adjoint system (5), the boundary conditions (6), and the solution of the switching system (7). The eigenfrequency is denoted as $\omega=\sqrt{K_{J} / M}$.

Since all system equations (row 3 ) are linear in $u$, the Pontryagin maximum principle leads to bang-bang control. The optimal switching functions are the terms of the particular Hamilton (row 4) that linearly depend on $u$. Together with its final conditions (row 5) the adjoint equation system (row 4) forms a final value problem.

For case A we obtain following solution for the relevant adjoint $\lambda_{1}$.

$$
\lambda_{1}=\omega \sin \left(\omega\left(t-t_{f}\right)\right)
$$

The switching law is therefore

$$
\dot{\theta}_{d}^{*}=\dot{\theta}_{\max } \operatorname{sgn}\left(\sin \left(\omega\left(t-t_{f}\right)\right)\right) .
$$

This rectangular function, which frequency is the resonance frequency of the joint has a phase shift that depends on $t_{f}$ in order to maximize the link side velocity at this particular time instant. Figure 3 depicts an example for the solution of the adjoint and system equation as well as the input. This result leads to the conclusion that with half period $t=\omega /(4 \pi)$ the link side velocity is doubled.

As for case A the optimal control trajectory of case B is derived also from Pontriyagin's maximum principle. The solution is again linear in $u$ and thus of bang-bang type. The switching times depend for case B on $\operatorname{sign}\left(\lambda_{2}\right)$, which is found to be 

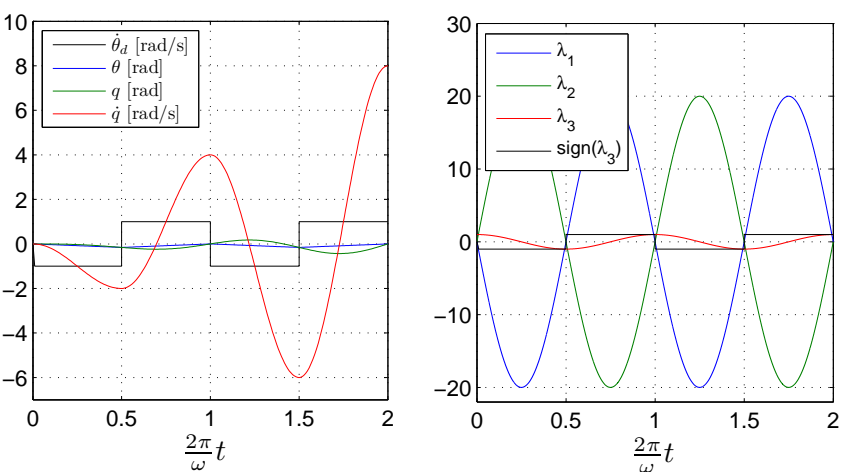

Fig. 3. Solution of the adjoint and system equations.

$$
\begin{aligned}
\lambda_{2}(t) & =\left(B^{2} K_{J} \mathrm{e}^{\frac{K_{P}\left(t-t_{f}\right)}{B}}-B^{2} K_{J} \cos \left(\omega\left(t-t_{f}\right)\right)\right. \\
& \left.-B K_{P} \sin \left(\omega\left(t-t_{f}\right)\right) \sqrt{K_{J} M}\right)\left(K_{P}^{2} M+K_{J} B^{2}\right)^{-1} .
\end{aligned}
$$

Compared to case A the switching condition consists of an additional trigonometric and exponential lag term. However, the principal structure remains the same.

For case $\mathrm{C}$ the solution is also similar to the previous ones, except for some additional trigonometric and exponential terms. Again, they do not alter the principal switching structure. The switching condition is

$$
\begin{aligned}
\lambda_{1}+\frac{K_{D}}{B} \lambda_{3} & =-\frac{K_{D} K_{J}^{2} B}{X 1} \cos \left(\omega\left(t-t_{f}\right)\right) \\
& +\frac{\left(K_{J} B K_{P}-K_{J} K_{D}{ }^{2}-K_{P}{ }^{2} M\right) \sqrt{K_{J} M}}{X 1} \sin \left(\omega\left(t-t_{f}\right)\right) \\
& +\frac{X 4}{X 1 X 2} \mathrm{e}^{\frac{\left(t-t_{f}\right)\left(X_{2}+K_{D}\right)}{{ }^{B}}}+\frac{X 3}{X 1 X 2} \mathrm{e}^{\frac{\left(t-t_{f}\right)\left(-x_{2}+K_{D}\right)}{{ }^{B}}}
\end{aligned}
$$

with

$$
\begin{aligned}
& \mathrm{X} 1=K_{J} K_{D}^{2} M+K_{J}^{2} B^{2}-2 K_{J} B K_{P} M+K_{P}^{2} M^{2} \\
& \mathrm{X} 2=\sqrt{K_{D}^{2}-4 K_{P} B} \\
& \mathrm{X} 3=1 / 2 K_{J} B\left(-2 K_{J} B K_{P}+2 K_{P}^{2} M+K_{J} K_{D} \sqrt{K_{D}^{2}-4 K_{P} B}+K_{J} K_{D}^{2}\right) \\
& \mathrm{X} 4=1 / 2 K_{J} B\left(2 K_{J} B K_{P}-2 K_{P}^{2} M+K_{J} K_{D} \sqrt{K_{D}^{2}-4 K_{P} B}-K_{J} K_{D}^{2}\right) .
\end{aligned}
$$

In order to complete the motor model, the feedback of the elastic joint torque shall be considered now (case D). Table 2 lists again all relevant equations and also the switching law. Unfortunately, we did not find an analytical solution for this system. Therefore, numerical methods have to be applied. Since the adjoints are not coupled with the system's differential equation they can e.g. be solved with the Runge-Kutta method via numerical integration.

A comparison of the different motor models is depicted in Fig. 4 , showing the dynamic response of $\dot{\theta}$ for $\dot{\theta}_{d}$, being the step function. Two main observations can be made: The significant switching time between PT1 and PT2 and the negligible influence of the elastic joint torque $\tau_{J}$ on the motor response of the PT2 model.

The main conclusions up to now are

- Motor dynamics do not influence the principal switching structure.

- Every delay element leads to a phase shift of the switching times.

- No analytical solution was found, when adding the influence of the elastic joint torque $\tau_{J}$.

- Insufficient motor dynamics lead to a saturation of the characteristic velocity increase curve (not described for brevity).

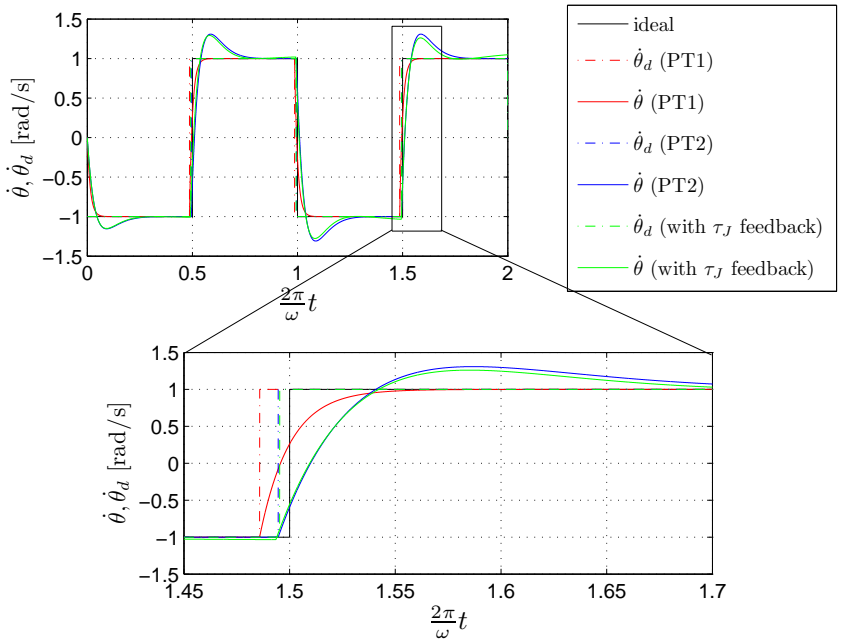

Fig. 4. Comparison of the different models.

In the next section we discuss the influence of an important real-world constraint of VIA joints: the elastic deflection limit $\varphi_{\max }$.

\section{CONSTRAINED DEFLECTION}

$\varphi_{\max }$ can be expressed as an inequality constraint on the difference of motor and link side position. Its second derivative incorporates the control variable. Thus, the order of the constraint is $q=2$ and one contact point exists.

$$
\begin{aligned}
& S^{(0)}:=(\theta-q)-\varphi_{\max } \leq 0 \\
& S^{(1)}:=(\dot{\theta}-\dot{q}) \leq 0 \\
& S^{(2)}:=(\ddot{\theta}-\ddot{q}) \leq 0
\end{aligned}
$$

The formulation of the optimal control problem with constraints is based on the model of case D. The Hamiltonian is extended by a term that incorporates new Lagrange multipliers $\boldsymbol{\mu}$. In total one obtains an 11 th order canonical system of differential equations with side constraints. For contact points the conditions given in Bryson and Ho [1975] count. This leads to a jump in the adjoint variables for the contact time $t_{b}$. Because $\frac{\partial S^{(2)}}{\partial x_{i}}=0$ and for choosing $\mu_{1}=0$ we may write

$$
\begin{aligned}
& \lambda_{2}\left(t_{b}^{+}\right)=\lambda_{2}\left(t_{b}^{-}\right)+\mu_{0} \frac{d S^{(0)}}{d x_{2}} \\
& \lambda_{4}\left(t_{b}^{+}\right)=\lambda_{4}\left(t_{b}^{-}\right)+\mu_{0} \frac{d S^{(0)}}{d x_{4}} .
\end{aligned}
$$

The concrete jumping conditions are

$$
\begin{aligned}
& \lambda_{2}\left(t_{b}^{+}\right)=\lambda_{2}\left(t_{b}^{-}\right)+\mu_{0} \\
& \lambda_{4}\left(t_{b}^{+}\right)=\lambda_{2}\left(t_{b}^{-}\right)-\mu_{0} .
\end{aligned}
$$

The additional trivial differential equation is

$$
\dot{\mu}_{0}=0 \text {. }
$$

The full system of equations can be solved with a numerical multiple-shooting method as e.g. described in Bulirsch and Stoer [1978], Carl-Cranz-Gesellschaft [1981].

Figure 5 depicts such a numerical solution of the multi point boundary value problem (MPBVP) obtained with 


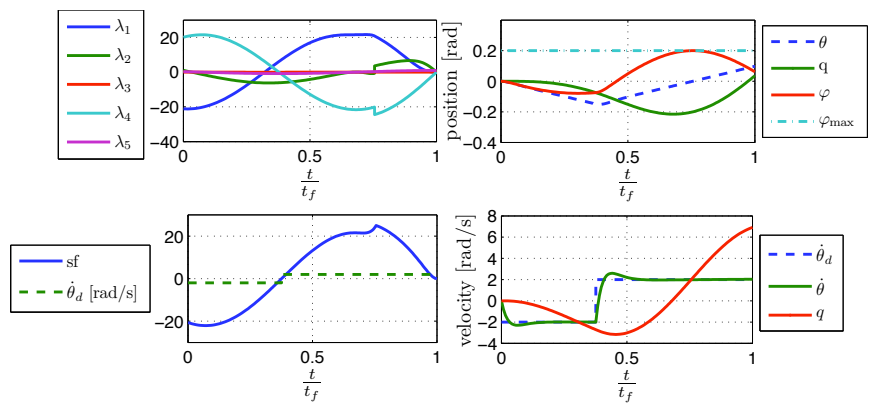

Fig. 5. Optimization with limited elastic deflection. $s f$ denotes the switching function.

the multiple-goal method implemented with the program BNDSCO Oberle [2001]. Important to notice is that for the constrained deflection case the optimization aims at the maximal elastic deflection (upper right). The optimal switching time is rather defined by keeping the constraints than resonant excitation.

In the next section we discuss to what extent the stiffness adjustment during motion contributes to an increase in maximum link side velocity.

\section{STIFFNESS ADJUSTMENT}

First, we take into consideration the influence of stiffness adjustment without a deflection constraint and then analyze the effect such limits have.

\subsection{Unconstrained deflection}

In order to elaborate the effect of stiffness adjustment, we choose the underlying model for this analysis to be the one of case A. The joint stiffness is now considered as an additional control input. Overall, the system equations are

$$
\begin{gathered}
\theta=\int \dot{\theta}_{d} d t \quad \text { with } \dot{\theta}_{\min } \leq \dot{\theta} \leq \dot{\theta}_{\max } \\
M \ddot{q}=K_{J}(t)(\theta-q) \quad \text { with } K_{J, \min } \leq K_{J}(t) \leq K_{J, \max },
\end{gathered}
$$

with $\mathbf{x}=\left[\begin{array}{lll}\theta & q & \dot{q}\end{array}\right]^{T}$ being the state vector and $\mathbf{u}=$ $\left[\begin{array}{ll}\dot{\theta}_{d} & K_{J}(t)\end{array}\right]^{T}$ the control input vector. The canonical system of differential equations is

$$
\begin{aligned}
& \dot{x}_{1}=u_{1} \\
& \dot{x}_{2}=x_{3} \\
& \dot{x}_{3}=\frac{u_{2}}{M}\left(x_{1}-x_{2}\right)
\end{aligned}
$$

$$
\begin{aligned}
& \dot{\lambda}_{1}=-\lambda_{3} \omega^{2} \\
& \dot{\lambda}_{2}=\lambda_{3} \omega^{2} \\
& \dot{\lambda}_{3}=-\lambda_{2} .
\end{aligned}
$$

The corresponding Hamiltonian can be derived as

$$
H(\mathbf{x}(t), \boldsymbol{\lambda}(t), \mathbf{u}(t), t)=\lambda_{1} u_{1}+\lambda_{2} x_{3}+\lambda_{3} \frac{u_{2}}{M}\left(x_{1}-x_{2}\right) .
$$

The Hamiltonian is linear in $u_{1}$ and $u_{2}$, leading directly to following switching laws.

$$
\begin{gathered}
\dot{\theta}_{d}^{*}=\left\{\begin{array}{cl}
\dot{\theta}_{\max }, & \lambda_{1}>0 \\
\dot{\theta}_{\min }, & \lambda_{1}<0 \\
\text { singular, } & \lambda_{1}=0
\end{array}\right. \\
K_{J, d}^{*}=\left\{\begin{array}{cc}
K_{J, \max }, & \lambda_{3} \frac{x_{1}-x_{2}}{M}>0 \\
K_{J, \min }, & \lambda_{3} \frac{x_{1}-x_{2}}{M}<0 \\
\text { singular, } & \lambda_{3} \frac{x_{1}-x_{2}}{M}=0
\end{array}\right.
\end{gathered}
$$

Due to the bang-bang structure of the desired stiffness the solution of the adjoints is similar to (9). However, this time a variable eigenfrequency characterizes the result ${ }^{3}$.

$$
\begin{aligned}
& \lambda_{1}=\sqrt{\frac{u_{2}}{M}} \sin \left(\sqrt{\frac{u_{2}}{M}}\left(t-t_{f}\right)\right) \\
& \lambda_{3}=\cos \left(\sqrt{\frac{u_{2}}{M}}\left(t-t_{f}\right)\right)
\end{aligned}
$$

For the present case two adjoints influence the switching condition. $\lambda_{1}$ determines the excitation of the system with $\dot{\theta}_{d}$ in resonance, depending on the current eigenfrequency. The stiffness switching function is characterized by two terms. First, the sign of the elastic deflection $\operatorname{sign}\left(x_{1}-x_{2}\right)$ and secondly, the switching function $\lambda_{3}$.

\subsection{Constrained deflection}

Based on Sec. 4 it is clear that the stiffness adjustment between maximal elastic deflection (maximum potential energy stored) and the time instant of maximal velocity (moment of launch) is critical. Therefore, we investigate the maximization of the Hamiltonian (29) during this particular time interval. The term containing the stiffness $u_{2}$ and the elastic deflection $\left(x_{1}-x_{2}\right)=(\theta-q)$ is to be maximized.

$$
\max \left\{\lambda_{3} \frac{u_{2}}{M}\left(x_{1}-x_{2}\right)\right\} .
$$

$\left(x_{1}-x_{2}\right)$ is always larger than zero between the moment of its maximal value and and launch. The maximal value will be achieved the earliest at $t_{f}-\frac{1}{2 \pi \omega}$. Due to the transversality condition $\partial h\left(\mathbf{x}\left(t_{f}\right)\right) / \partial x_{3}=\partial \dot{q}\left(t_{f}\right) / \partial \dot{q}=1$ the last adjoint $\lambda_{3}$ reaches its maximal value $\lambda_{3}=1$ at $t_{f}$ (see (33)). Furthermore, it changes its sign also at a quarter of the periodicity before the launch time. The switching function $\lambda_{3}$ is consequently positive in the considered time interval. This leads, according to the maximum principle, to maximizing the stiffness (see (31)) towards the moment of launch.

$$
K_{J}^{*}=K_{J, \max } \quad t_{b} \leq t \leq t_{f}
$$

Up to now, we assumed that the stiffness trajectory before the boundary point does not influence the end velocity. Therefore, it seems reasonable to set the stiffness to its maximum value during the throwing trajectory without additionally adjusting the stiffness. However, from a practical point of view it can be necessary to start the motion at low stiffness adjustment and enlarge it towards the launch time. This can have three main reasons:

- The motor dynamics is not sufficient to excite the joint at maximum stiffness at the corresponding eigenfrequency.

- The motor power is not sufficient to deflect the joint with an adequately low number of switching cycles.

- Limits on the elastic deflection can lead to higher energy storage for lower stiffness ranges due to higher possible deflection than for higher stiffness presets.

The last aspect can be explained with Fig. 6 and is caused by the implemented working principle of the VIA mechanism. The left figure shows two different linear stiffness curves for which the maximum deflection is constant for

3 Pease note that the eigenfrequency is not continuously varying, but switching between its minimum and maximum value. 

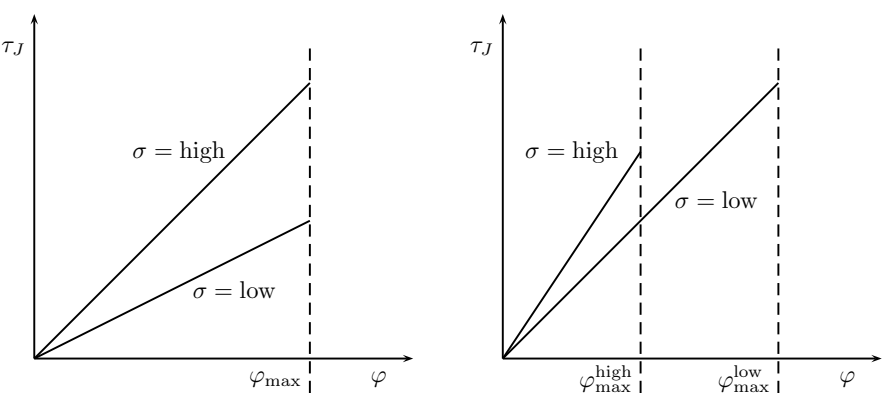

Fig. 6. Deflection limits $\varphi_{\max }$ for different stiffness presets $\sigma$. The left figure shows a design, where $\varphi_{\max }$ is constant for every $\sigma$ and the right one depicts a functional relationship between $\varphi_{\max }$ and $\sigma$.

all presets. On the right one characteristics are depicted, where a functional relationship between maximum deflection and stiffness preset exists.

First, let us discuss the former. According to the maximum principle the Hamiltonian is maximized through the entire motion process and therefore the joint stiffness as well. Consequently, the potential energy stored in the joint elasticity is maximized for every deflection. This induces that it is not optimal to change the stiffness, on the contrary, it reduces the achievable link velocity.

For the latter characteristics the maximum elastic energy that can be stored depends on the deflection. For large deflection a soft preset and for small deflection a stiff one are to be preferred. Maximization of joint torque is therefore directly coupled with adjusting stiffness along the admissible deflection.

Next, we discuss the analysis for a concrete joint design and present various experimental results.

\section{ANALYSIS FOR THE QA-JOINT}

In this section we apply the elaborated insights to a concrete VIA design, the DLR QA-Joint.

\subsection{Without stiffness adjustment}

For the QA-Joint, Eiberger et al. [2010], the elastic joint torque $\tau_{J}$ is defined as

$$
\tau_{J}=40\left(e^{15(\varphi-\sigma)}-e^{15(-\varphi-\sigma)}\right)
$$

so the mapping $\sigma \rightarrow K_{J}=\partial \tau_{J} / \partial q$ is a nonlinear function. With the state vector $\mathbf{x}^{T}=\left[\begin{array}{lllll}\theta_{d} & \theta & \dot{\theta} & q & \dot{q}\end{array}\right], u=\dot{\theta}_{d}$ and initial conditions $\mathbf{x}^{T}(0)=\left[\begin{array}{lllll}0 & 0 & 0 & 0 & 0\end{array}\right]$ we obtain the following system of differential equations when assuming elastic torque feedback and PT2 motor behavior.

$$
\dot{x}_{1}=u \quad \dot{x}_{2}=x_{3} \quad \dot{x}_{3}=\frac{1}{B}\left(\tilde{\tau}_{m}-\tau_{J}\right) \quad \dot{x}_{4}=x_{5} \quad \dot{x}_{5}=\frac{\tau_{J}}{M}(37)
$$

$\tilde{\tau}_{m}$ denotes the bounded motor torque

$$
\tilde{\tau}_{m}=\left\{\begin{array}{cc}
\tau_{m, \max } & \tau_{m, d} \geq \tau_{m}^{\max } \\
\tau_{m, d} & \tau_{m}^{\min }<\tau_{m, d}<\tau_{m}^{\max } \\
\tau_{m, \min } & \tau_{m, d} \leq \tau_{m, d}^{\min },
\end{array}\right.
$$

with $\tau_{m, d}=K_{D}\left(u-x_{3}\right)+K_{P}\left(x_{1}-x_{2}\right)$ being the desired motor torque from the PD controller. The Hamiltonian is

$$
H(.)=\lambda_{1} u+\lambda_{2} x_{3}+\lambda_{3} \frac{1}{B}\left(\tilde{\tau}_{m}-\widetilde{\tau}_{J}(\sigma)\right)+\lambda_{4} x_{5}+\lambda_{5} \frac{1}{M} \tau_{J}(\sigma) .
$$

The optimal control problem to be solved consists of a system of differential equation of 11th order (adjoint and system equations), including the additional trivial differential equation if taking into account the elastic deflection limit with one boundary point, see Sec. 4 . The nonlinearity causes a coupling of the adjoint and state equations, leading to a MPBVP with separated initial and end conditions for the canonical system of differential equations ${ }^{4}$. The limits of motor torque eventually lead to a necessary formulation of boundary control. Solving this problem with multi-goal methods turned out to be very unstable. This is because on the one side for $n$ nodes $5 n$ starting conditions need to be estimated and their deviation from the solution is highly influencing the convergence of the method. Furthermore, a physical interpretation of the adjoint variables is also not given. Thus, the estimation of their start values, which would lead to a solution is not straight forward.

A possibility to solve this optimization is a parameter estimation method by utilizing the information that the optimal control trajectory shows bang-bang behavior (which comes from the linear occurrence of the input into the state equation). This is also independent from the limit in motor torque $\tilde{\tau}_{m}$ (see (38)), as the principal structure of the Hamiltonian remains the same regardless of the saturation $^{5}$ :

$$
\begin{aligned}
\widetilde{H}(\boldsymbol{\lambda}(t), u(t)) & =\left(\lambda_{1}+\lambda_{3} \frac{K_{D}}{B}\right) u, \quad \tau_{m, \min }<\tau_{m, d}<\tau_{m, \max } \\
\widetilde{H}(\boldsymbol{\lambda}(t), u(t)) & =\lambda_{1} u, \quad\left(\tau_{m, d}<\tau_{m, \min }\right) \vee\left(\tau_{m, d}>\tau_{m, \max }\right)
\end{aligned}
$$

The parameter to be estimated is the switching time. The optimization is carried out by multiple solving of the system equations with the jumping times in the control variable being timely varied via appropriate optimization. The used algorithm is the Nelder-Mead simplex downhill method with the following optimization criterion.

$$
J=-\dot{q}+J_{p}
$$

$$
J_{p}=\left\{\begin{array}{cc}
0 & \varphi_{\min } \leq \varphi \leq \varphi_{\max } \\
\exp \left(|\varphi|-\varphi_{\max }\right) & |\varphi|>\varphi_{\max }
\end{array}\right.
$$

Complying with the constraints is ensured with penalty term $J_{p}$.

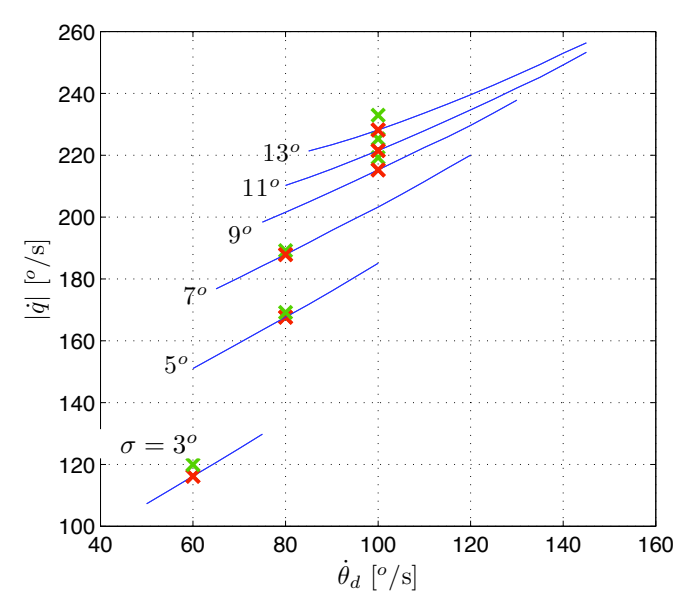

Fig. 7. Final link velocity as a function of motor velocity.

\footnotetext{
4 The adjoint system is given in Appendix A.

5 Please note that only the relevant term of the Hamiltonian is
} shown, which linearly depends on $u$. 


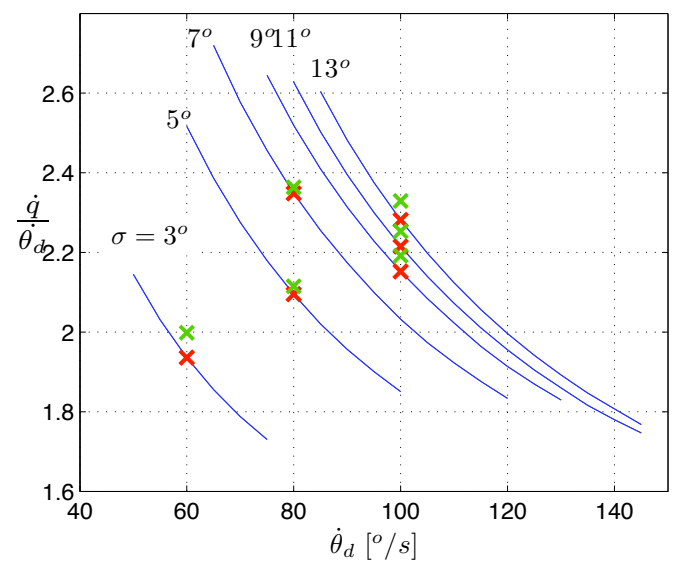

Fig. 8. Relative final link side velocity as a function of motor velocity.

Under the premise of achieving maximal deflection with one switching cycle (throwing with striking out once), a limited velocity range for the position motor complies. On the one hand, a minimum velocity for achieving the maximal deflection is needed and on the other side there exists a maximum velocity at which the constraint still can be ensured. The simulation results are depicted in Fig. 7 and Fig. 8. The red marked points on the theoretical graphs were experimentally verified (green crosses).

Figure 7 shows the absolute achievable final velocity as a function of commanded motor velocity characterized by the almost linear relationship. This induces a continuous velocity increase with stored potential energy. Furthermore, it becomes clear that too low elasticity leads to a degradation of achievable link velocity. The relative velocity increase with respect to the motor velocity at final time is depicted in Fig. 8. If this relation is considered as the speed gain ${ }^{6}$ of the elastic mechanism, it can be stated that it degrades with increasing motor velocity and increasing stiffness. As already explained, it is necessary to drive with higher motor velocities to achieve the maximum deflection for low stiffness. For the QA-Joint the largest speed gain can be obtained at $\theta_{d}=65 \mathrm{o} / \mathrm{s}$ and moderate stiffness. This is equivalent to an efficiency of 2.7 .

In Figure 9 the time courses of measurements and simulations for high and low stiffness presets are shown. The relevant variables are the link side velocity, deflection, and the elastic joint torque.

- link velocity (left):

The trajectory of the link velocity shows very good consistency with the simulation. At final time the velocity is approximately twice the motor velocity. The deviation in joint torque are almost not reflected in the velocity profile.

\section{- deflection (middle):}

In contrast to the simulation a slight exceedance of the deflection constraints can be observed in the lower row. This is mainly due to the variance in the identified stiffness and friction parameters, calibration errors, and simplified assumptions for the friction model.

- joint torque (right):

The principal time course of the joint torque confirms

6 Please note that we consider the speed gain to be a relevant quantity as it relates the achievable link side velocity in direct relation to the maximum desired motor velocity, i.e. it directly relates the relative benefit that one may obtain in principle.
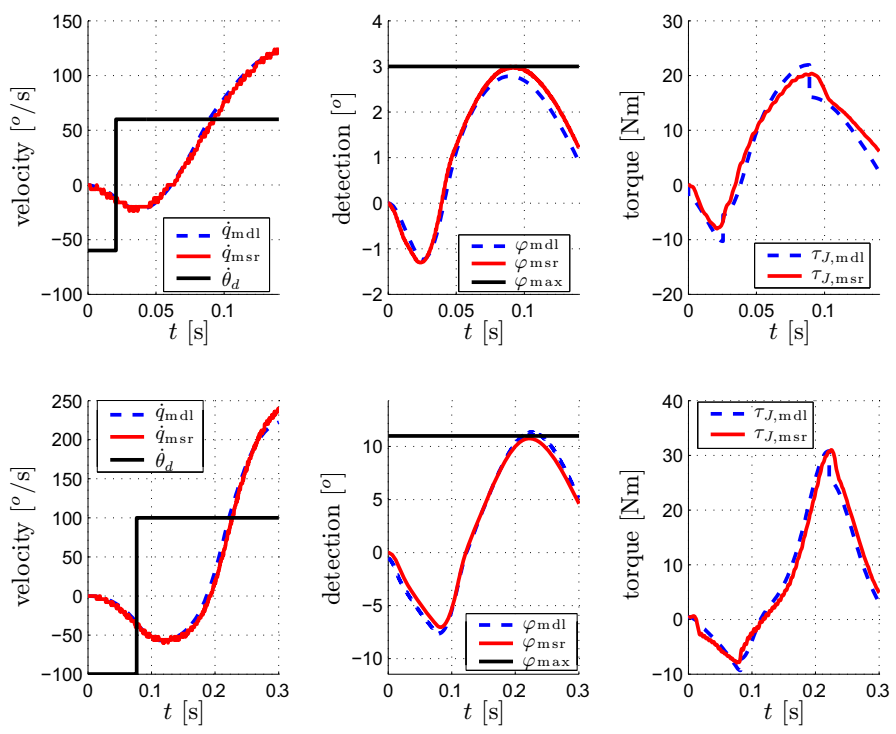

Fig. 9. Comparison of simulation and measurements for different stiffness presets. The upper row shows the motion for $\dot{\theta}_{d}=60^{\circ} / \mathrm{s}$ and $\sigma=3^{\circ}$. The lower row depicts the results for $\dot{\theta}_{d}=100^{\circ} / \mathrm{s}, \sigma=11^{\circ}$.

the joint model with respect to the identification of stiffness and friction. The discontinuities in the simulation are caused by the Coulomb friction model during change of direction.

Next, we discuss the effect of stiffness adjustment for the QA-Joint.

\subsection{Stiffness adjustment}

For the stiffness adjustment during the motion there are also some conclusions to be drawn. For the linear joint stiffness it was shown that the relation between stiffness and deflection is critical, see Sec. 5.1. For the QA-Joint this constraint is formally defined as

$$
\sigma \geq \varphi \quad \sigma \in\left[3^{o} 15^{\circ}\right]
$$

For maximizing the Hamiltonian (39), following term is considered, which explicitly depends on the stiffness adjustment $\sigma$.

$$
\widetilde{H}(\boldsymbol{\lambda}(t), \mathbf{x}(t), \sigma(t))=\underbrace{\left(\lambda_{5} \frac{1}{M}-\lambda_{3} \frac{1}{B}\right)}_{\lambda^{*}} \tau_{J}(\sigma)
$$

As assumed in Sec. 5.1 only a stiffening during the relaxation phase is essential. Thus, the sign of $\dot{\varphi}$ does not change. In Appendix A it is shown that $\lambda^{*} \geq 0$ holds during the entire adjustment phase. Therefore, $\tau_{J}$ has to be maximized.

$$
\tau_{J}=\frac{1}{e^{15 \sigma}}\left[\left(a_{S}-a_{R}\right) e^{15\left(x_{2}-x_{4}\right)}-\left(b_{S}+b_{R}\right) e^{15\left(-x_{2}+x_{4}\right)}\right]
$$

The maximization of the elastic torque in turn necessitates the maximization of stiffness, respectively a minimization of $\sigma$ at every time instant. Taking (44) into account the optimal stiffness trajectory is

$$
\sigma^{*}=\left\{\begin{array}{l}
3 ; \quad \varphi \leq 3 \\
\varphi ; 3<\varphi<15 .
\end{array} \quad t_{b} \leq t \leq t_{f}\right.
$$

This means that the acceleration torque has to be sustained during relaxation as long as possible. From an energy point of view the stiffness adjuster injects additional 


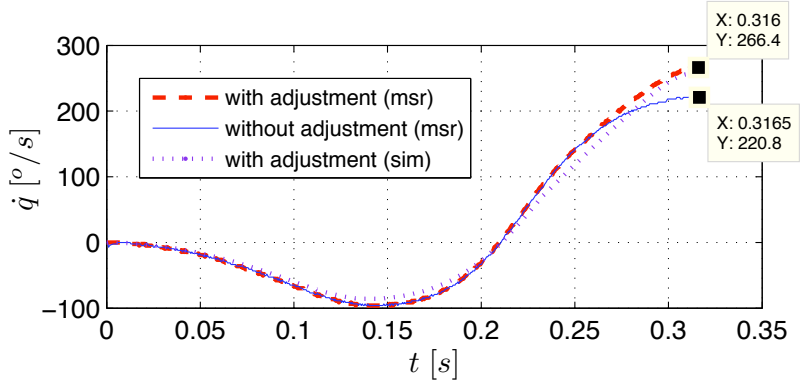

Fig. 10. Link side velocity with stiffness adjustment.

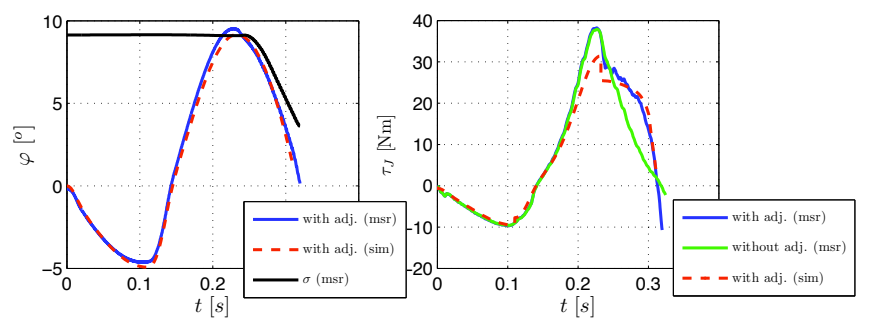

Fig. 11. Deflection (left) and joint torque (right) with stiffness adjustment.

energy such that the joint maximally stores potential energy for a certain deflection. The potential energy that can be converted into kinetic energy is therefore maximized at the same time.

The according experimental verification is depicted in Fig. 10 and Fig. 11. For a moderate stiffness preset $\sigma=9^{\circ}$ the achieved link velocity is $266^{\circ} / \mathrm{sec}$., which is approximately $20 \%$ higher than without adjustment. From Figure 11 (left) it can be observed that adjusting the stiffness according to (47) is not fully achieved due to too little dynamics of the stiffness motor ${ }^{7}$. Nonetheless, a significant velocity increase is observed here as well. Compared to the constant elasticity case the joint torque shows an increase from the moment of adjustment on, confirming the theoretical requirement to maximize the sustaining torque during relaxation phase.

\section{CONCLUSION}

In this paper we developed a theoretically sound concept to achieve an optimal speed gain for Variable Impedance Actuators based on optimal control theory. We analyzed models of increasing complexity to identify the main factors determining the achievable performance and incorporated the most important real-world constraints into the analysis. Furthermore, we verified the schemes on the DLR QA-Joint, a novel VSA prototype, which is one of the basis mechanisms for the anthropomorphic DLR hand arm system. Our future work will deal with the extension to the N-DoF case.

\section{ACKNOWLEDGEMENTS}

We would like to thank Felix Huber for his valuable support.

\section{REFERENCES}

Alin Albu-Schäffer, Christian Ott, and Gerd Hirzinger. A unified passivity-based control framework for position,

7 Please note that the stiffness adjuster is assumed to show ideal behavior for the simulation. torque and impedance control of flexible joint robots. The Int. J. of Robotics Research, 26:23-39, 2007.

Alin Albu-Schäffer, Oliver Eiberger, Markus Grebenstein, Sami Haddadin, Christian Ott, Thomas Wimböck, Sebastian Wolf, and Gerd Hirzinger. Soft robotics: From torque feedback controlled lightweight robots to intrinsically compliant systems. IEEE Robotics and Automation Mag.: Special Issue on Adaptable Compliance/Variable Stiffness for Robotic Applications, 15(3): $20-30,2008$.

Arthur E. Bryson and Yu-Chi Ho. Applied optimal control. Hemisphere Publ. Corp., rev. print. edition, 1975. ISBN 0-470-26774-7, 0-470-11481-9.

R. Bulirsch and J. Stoer. Einführung in die numerische Mathematik 2. Springer-Verlag, 1978. (German).

Carl-Cranz-Gesellschaft. Optimierungsverfahren- Software und praktische Anwendungen, 1981. (German).

Oliver Eiberger, Sami Haddadin, Michael Weis, Alin AlbuSchäffer, and Gerd Hirzinger. On joint design with intrinsic variable compliance: Derivation of the DLR QA-joint. IEEE Int. Conf. on Robotics and Automation (ICRA2010), Anchorage, Alaska, pages 1687-1694, 2010 .

M. Grebenstein and P. van der Smagt. Antagonism for a highly anthropomorphic hand-arm system. Advanded Robotics, 22(1):39-55, 2008.

S. Haddadin, T. Laue, U. Frese, and G. Hirzinger. Foul 2050: Thoughts on physical interaction in human-robot soccer. In IEEE/RSJ Int. Conf. on Intelligent Robots and Systems (IROS2007), San Diego, USA, pages 32433250, 2007.

I.P. Herman. Physics of the Human Body. Springer Verlag, 2007.

H.J. Oberle. BNDSCO - A Program for the Numerical Solution of Optimal Control Problems, October 2001. Hamburger Beiträge zur angewandten Mathematik, Bericht 36, Reihe B.

M. Okada, S. Ban, and Y. Nakamura. Skill of compliance with controlled charging/discharging of kinetic energy. In IEEE Int. Conf. on Robotics and Automation (ICRA2002), Washington, USA, pages 2455-2460, 2002.

D. Paluska and H. Herr. The effect of series elasticity on actuator power and work output: Implications for robotic and prosthetic joint design. Robotics and Autonomous Systems, 54:667-673, 2006.

H. Schempf, C. Kraeuter, and M. Blackwell. ROBOLEG: A robotic soccer-ball kicking leg. In IEEE Int. Conf. on Robotics and Automation (ICRA1995), Nagoya, Aichi, Japan, volume 2, pages 1314 - 1318, 1995.

Ronald van Ham, Thomas Sugar, Bram Vanderborgth, Kevin Hollander, and Dirk Lefeber. Compliant actuator designs: Review of actuators with passive adjustable compliance/controllable stiffness for robotic applications. IEEE Robotics and Automation Mag., 16(3): 81-94, 2009.

B. Vanderborght, B. Verrelst, R. Van Ham, M. Van Damme, D. Lefeber, B.M.Y. Duran, and P. Beyl. Exploiting natural dynamics to reduce energy consumption by controlling the compliance of soft actuators. Int. Journal of Robotics Research, 25(4):343-358, 2006. ISSN 0278-3649.

S. Wolf and G. Hirzinger. A new variable stiffness design: Matching requirements of the next robot generation. IEEE Int. Conf. on Robotics and Automation (ICRA 2008), Pasadena, USA, pages 1741-1746, 2008.

J. Yamaguchi, S. Inoue, D. Nishino, and A. Takanishi. Development of a bipedal humanoid robot having antagonistic driven joints and three DOF trunk. In IEEE/RSJ Int. Conf. on Intelligent Robots and Systems (IROS1998), Victoria, B.C., Canada, pages 96- 


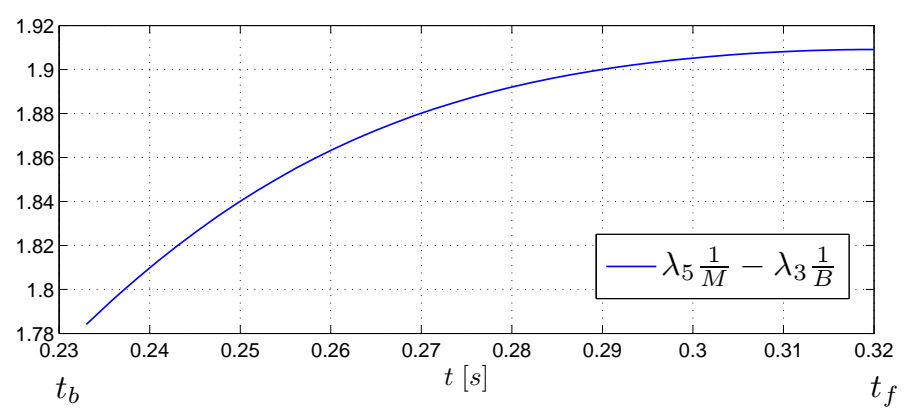

Fig. A.1. Solution of the switching function with stiffness adjustment.

101, 1998a.

J. Yamaguchi, D. Nishino, and A. Takanishi. Realization of dynamic biped walking varying joint stiffness using antagonistic driven joints. In IEEE Int. Conf. on Robotics and Automation (ICRA1998), Leuven, Belgium, pages 2022-2029, 1998b.

\section{Appendix A. SOLVING THE ADJOINT EQUATIONS}

In order to confirm the assumption $\lambda^{*} \geq 0$ for the experiment carried out in Sec. 6.2, the adjoint equations have to be solved for the time interval of stiffness adjustment. Since they do not show discontinuities they can be solved numerically as a final value problem by utilizing the already optimized solution of the state equations.

The solution of the adjoint equation systems in the time interval $\left[\begin{array}{ll}t_{b} & t_{f}\end{array}\right]$ gives the confirmation that the stiffness adjustment presented in Sec. 4 is indeed satisfying optimal control theory. For this, the switching function $\lambda^{*}$ has to have positive sign in this interval. The system of differential equation for the adjoints is

$$
\begin{aligned}
\dot{\lambda}_{1}= & -\lambda_{3} \frac{1}{B} K_{P} \\
\dot{\lambda}_{2}= & \lambda_{3} \frac{1}{B}\left(\left(b_{S}-b_{R}\right) \exp (15(\varphi-\sigma))\right. \\
& \left.-\left(a_{s}-a_{R}\right) \exp (15(\varphi-\sigma))+K_{P}\right) \\
\dot{\lambda}_{3}= & -\lambda_{2}+\lambda 3 \frac{K_{P}}{B} \\
\dot{\lambda}_{4}= & \left(\lambda_{5} \frac{1}{M}+\lambda_{3} \frac{1}{B}\right)\left(\left(b_{S}-b_{R}\right) \exp (15(\varphi-\sigma))\left(a_{s}-a_{R}\right) \exp (15(\varphi-\sigma))\right) \\
& +\left(a_{s}\right) \\
\dot{\lambda}_{5}= & -\lambda_{4},
\end{aligned}
$$

where $\varphi=x_{2}-x_{4}$. With final values $\boldsymbol{\lambda}^{T}\left(t_{f}\right)=\left[\begin{array}{lllll}0 & 0 & 0 & 0 & 1\end{array}\right]$ the problem can be formulated as final value problem and e.g. be solved with the Runge-Kutta method. Figure A.1 depicts the solution of the switching function $\lambda^{*}=\lambda_{5} \frac{1}{M}-$ $\lambda_{3} \frac{1}{B}$, showing the positive sign over the relevant time interval. 